\title{
Matrix Tensor Product Approach to the Equivalence of Multipartite States under Local Unitary Transformations
}

\author{
X. H. Gao ${ }^{1}$, S. Alberverio ${ }^{2}$, S. M. Fei ${ }^{1,2,3}$, Z.X. Wang ${ }^{1}$ \\ ${ }^{1}$ Department of Mathematics, Capital Normal University, Beijing 100037 \\ ${ }^{2}$ Institut für Angewandte Mathematik, Universität Bonn, D-53115 \\ ${ }^{3}$ Max-Planck-Institute for Mathematics in the Sciences, 04103 Leipzig
}

\begin{abstract}
The equivalence of multipartite quantum mixed states under local unitary transformations is studied. A criterion for the equivalence of non-degenerate mixed multipartite quantum states under local unitary transformations is presented.
\end{abstract}

PACS numbers: 03.67.-a, 02.20.Hj, 03.65.-w

Quantum entangled states are playing fundmental roles in quantum information processing such as quantum computation, quantum teleportation, dense coding, quantum cryptographic schemes quantum error correction, entanglement swapping, and remote state preparation (RSP) etc.. However the theory of quantum entanglement is still far from being satisfied. To quantify the degree of entanglement a number of entanglement measures have been proposed for bipartite states. Most of these proposed measures of entanglement involve extremizations which are difficult to handle analytically. For multipartite case how to give a well defined measure is still under discussion. For general mixed states till now we don't even have an operational criterion to verify whether a state is separable or not. As a matter of fact, the degree of entanglement of a multipartite quantum system remains invariant under local unitary transformations of every subsystems. Therefore the quantum states can be classified according to the local unitary transformations. Nevertheless an explicit picture of the orbits (geometry and topology) under such transformations is not yet ready. We even don't have a general (operational) criterion to verify if two mixed states are equivalent or not under local unitary transformations.

One approach in dealing with the equivalence of quantum states under local unitary transformations is to find the complete set of invariants under local unitary transformations. Two states are equivalent under local unitary transformations if and only if they have the same values of all these invariants. The method developed in $[1,2]$, in principle, allows one to compute all the invariants of local unitary transformations, though in general it is not operational. The invariants for general two-qubit and three qubits systems have been 
studied in [3, 4]. In [5] a complete set of invariants is presented for bipartite generic mixed states. In [6] a complete set of invariants under local unitary transformations is presented for rank-2 and multiplicity free mixed (bipartite) states. The invariants for pure tripartite states are also studied in $[7,8]$.

In [9] another approach is presented in investigating the equivalence problem in terms of fixed point subgroup and tensor decomposability of certain matrices. The problem is reduced to verify whether a certain matrix is rank one or not. A criterion for the equivalence of two non-degenerate mixed bipartite states under local unitary transformations has been presented.

It is rather difficult to deal with the equivalence problem of multipartite states in terms of invariants approach. The number of invariants increases quickly when the subsystems increase. Nevertheless, we find that the approach in [9] can be easily generalized to multipartite case. In this note we investigate the equivalence of multipartite states under local unitary transformations by using the method in [9]. An operational criterion for the equivalence of non-degenerate mixed multipartite states under local unitary transformations is presented.

We first consider tripartite case. Let $H_{1}$ (resp. $H_{2}$ and $H_{3}$ ) be an $M$ (resp. $N$ and $P$ )-dimensional complex Hilbert space, with $\left|e_{i}\right\rangle, i=1, \ldots, M\left(\operatorname{resp} .\left|f_{j}\right\rangle, j=1, \ldots, N\right.$ and $\left.\left|g_{k}\right\rangle, k=1, \ldots, P\right)$, as an orthonormal basis. A general pure state on $H_{1} \otimes H_{2} \otimes H_{3}$ is of the form

$$
|\Psi\rangle=\sum_{i=1}^{M} \sum_{j=1}^{N} \sum_{k=1}^{P} a_{i j k}\left|e_{i}\right\rangle \otimes\left|f_{j}\right\rangle \otimes\left|g_{k}\right\rangle, \quad a_{i j k} \in \mathbb{C}
$$

with the normalization $\sum_{i=1}^{M} \sum_{j=1}^{N} \sum_{k=1}^{P} a_{i j k} a_{i j k}^{*}=1$ ( $*$ denoting complex conjugation). A tripartite quantum mixed state on $H_{1} \otimes H_{2} \otimes H_{3}$ is described by a density matrix $\rho$ which can be decomposed according to its eigenvalues and eigenvectors: $\rho=\sum_{i=1}^{M N P} \lambda_{i}\left|\nu_{i}\right\rangle\left\langle\nu_{i}\right|$, where $\lambda_{i}$ are the eigenvalues and $\left|\nu_{i}\right\rangle, i=1, \ldots, M N P$, the corresponding eigenvectors of the form (1).

Two density matrices $\rho$ and $\rho^{\prime}$ are said to be equivalent under local unitary transformations if there exist unitary operators $U_{1}$ on $H_{1}, U_{2}$ on $H_{2}$ and $U_{3}$ on $H_{3}$ such that

$$
\rho^{\prime}=\left(U_{1} \otimes U_{2} \otimes U_{3}\right) \rho\left(U_{1} \otimes U_{2} \otimes U_{3}\right)^{\dagger} .
$$

For a Hermitian matrix $A$ on $H_{1} \otimes H_{2} \otimes H_{3}$, the set of commuting matrices $B$ such that $A B=B A$ is called the commutant of $A$, denoted as $C(A)$. Obviously $C(A)$ is a subalgebra. We call the set of unitary matrices $U$ such that $U A=A U$ the fixed point subgroup of $A$, denoted as $C_{U}(A)$, which is a subgroup of the unitary group of all unitary matrices. In the following we say that a matrix $V$ on $H_{1} \otimes H_{2} \otimes H_{3}$ is tensor decomposable if it can be written as $V=V_{1} \otimes V_{2} \otimes V_{3}$ for $V_{1} \in \operatorname{End}\left(H_{1}\right), V_{2} \in \operatorname{End}\left(H_{2}\right), V_{3} \in \operatorname{End}\left(H_{3}\right)$.

If two density matrices $\rho$ and $\rho^{\prime}$ are equivalent under local unitary transformations, they must have the same set of eigenvalues $\lambda_{i}, i=1, \ldots, n$. Let $X$ and $Y$ be the unitary matrices that diagonalize $\rho$ and $\rho^{\prime}$ respectively,

$$
\rho=X \Lambda X^{\dagger}, \quad \rho^{\prime}=Y \Lambda Y^{\dagger},
$$

where $\Lambda=\operatorname{diag}\left(\lambda_{1}, \lambda_{2}, \ldots, \lambda_{M N P}\right)$. 
[Lemma 1]. Let $G$ be the fixed point unitary subgroup associated with $\rho$. Then $\rho^{\prime}$ is equivalent to $\rho$ under local unitary transformations if and only if the coset $G X Y^{\dagger}$ contains a unitary tensor decomposable matrix.

[Proof]. Suppose $\rho^{\prime}=\left(U_{1} \otimes U_{2} \otimes U_{3}\right) \rho\left(U_{1} \otimes U_{2} \otimes U_{3}\right)^{\dagger}$, then $\rho^{\prime}=Y \Lambda Y^{\dagger}=Y X^{\dagger} \rho X Y^{\dagger}$. Hence $Y X^{\dagger} \rho X Y^{\dagger}=\left(U_{1} \otimes U_{2} \otimes U_{3}\right) \rho\left(U_{1} \otimes U_{2} \otimes U_{3}\right)^{\dagger}$, or

$$
\left(U_{1} \otimes U_{2} \otimes U_{3}\right)^{\dagger} Y X^{\dagger} \rho=\rho\left(U_{1} \otimes U_{2} \otimes U_{3}\right)^{\dagger} Y X^{\dagger} .
$$

That is, $\left(U_{1} \otimes U_{2} \otimes U_{3}\right)^{\dagger} Y X^{\dagger} \in C_{U}(\rho)$, and $C_{U}(\rho) X Y^{\dagger}=Y X^{\dagger} C_{U}(\rho)$ contains a unitary tensor decomposable element $U_{1} \otimes U_{2} \otimes U_{3}$.

Conversely, assume $G X Y^{\dagger}$ contains a tensor decomposable element $U_{1} \otimes U_{2} \otimes U_{3}$. We have then $U X Y^{\dagger}=U_{1} \otimes U_{2} \otimes U_{3}, U \rho=\rho U$ and $U$ is unitary. Therefore

$$
\begin{aligned}
\rho^{\prime} & =Y \Lambda Y^{\dagger}=\left(U_{1} \otimes U_{2} \otimes U_{3}\right)^{\dagger} U X \Lambda X^{\dagger} U^{\dagger}\left(U_{1} \otimes U_{2} \otimes U_{3}\right) \\
& =\left(U_{1} \otimes U_{2} \otimes U_{3}\right)^{\dagger} U \rho U^{\dagger}\left(U_{1} \otimes U_{2} \otimes U_{3}\right)=\left(U_{1} \otimes U_{2} \otimes U_{3}\right)^{\dagger} \rho\left(U_{1} \otimes U_{2} \otimes U_{3}\right)
\end{aligned}
$$

Let $Z$ be an a matrix on $H_{1} \otimes H_{2} \otimes H_{3}$. If we view $Z$ as a matrix on spaces $H_{1}$ and $H_{2} \otimes H_{3}$, it is an $M \times M$ block matrix with each block of size $N P \times N P$. Its realigned matrix $\tilde{Z}_{1 \mid 23}$ is defined by

$$
\tilde{Z}_{1 \mid 23}=\left[\operatorname{vec}\left(Z_{11}\right), \cdots, \operatorname{vec}\left(Z_{1 M}\right), \cdots, \operatorname{vec}\left(Z_{M 1}\right), \cdots, \operatorname{vec}\left(Z_{M M}\right)\right]^{t}
$$

Taking $Z$ as a matrix on spaces $H_{1} \otimes H_{2}$ and $H_{3}$, we have that $Z$ is an $M N \times M N$ block matrix with each block of size $P \times P$, and the realigned matrix $\tilde{Z}_{12 \mid 3}$ is of the form

$$
\tilde{Z}_{12 \mid 3}=\left[\operatorname{vec}\left(Z_{11}\right), \cdots, \operatorname{vec}\left(Z_{1 M N}\right), \cdots, \operatorname{vec}\left(Z_{M N 1}\right), \cdots, \operatorname{vec}\left(Z_{M N M N}\right)\right]^{t}
$$

where for any $M \times N$ matrix $A$ with entries $a_{i j}, \operatorname{vec}(A)$ is defined to be

$$
\operatorname{vec}(A)=\left[a_{11}, \cdots, a_{1 N}, a_{21}, \cdots, a_{2 N}, \cdots, a_{M 1}, \cdots, a_{M N}\right]^{t} .
$$

The above operation of realignment could be defined in an alternative way,

$$
\left(\tilde{Z}_{1 \mid 23}\right)_{i m, j k n p}=(Z)_{i j k, m n p}, \quad\left(\tilde{Z}_{12 \mid 3}\right)_{i j m n, k p}=(Z)_{i j k, m n p}
$$

It is shown that a matrix $V$ can be expressed as the tensor product of two matrices $V_{1}$ and $V_{2}, V=V_{1} \otimes V_{2}$, if and only if [10]

$$
\tilde{V}=\operatorname{vec}\left(V_{1}\right) \operatorname{vec}\left(V_{2}\right)^{t}
$$

Moreover [7], for an $M N \times M N$ unitary matrix $U$, if $U$ is a unitarily decomposable matrix, then the rank of $\tilde{U}$ is one, $r(\tilde{U})=1$. Conversely if $r(\tilde{U})=1$, there exists an $M \times M$ matrix $U_{1}$ and an $N \times N$ matrix $U_{2}$, such that $U=U_{1} \otimes U_{2}$ and

$$
U_{1} U_{1}^{\dagger}=U_{1}^{\dagger} U_{1}=k^{-1} I_{M}, \quad U_{2} U_{2}^{\dagger}=U_{2}^{\dagger} U_{2}=k I_{N}
$$

where $I_{N}\left(\right.$ resp. $\left.I_{M}\right)$ denotes the $N \times N($ resp. $M \times M)$ identity matrix, $k>0$, and $U$ is a unitary tensor decomposable matrix. 
[Lemma 2]. For an $M N P \times M N P$ unitary matrix $U$ on $H_{1} \otimes H_{2} \otimes H_{3}, U$ is a unitary decomposable matrix if and only if the ranks of $\tilde{U}_{1 \mid 23}, \tilde{U}_{12 \mid 3}$ are one, i.e., $r\left(\tilde{U}_{1 \mid 23}\right)=r\left(\tilde{U}_{12 \mid 3}\right)=$ 1.

[Proof]. Suppose $U=U_{1} \otimes U_{2} \otimes U_{3}$, then $\tilde{U}_{1 \mid 23}=\operatorname{vec}\left(U_{1}\right) \operatorname{vec}\left(U_{2} \otimes U_{3}\right)^{t}, \tilde{U}_{12 \mid 3}=\operatorname{vec}\left(U_{1} \otimes\right.$ $\left.U_{2}\right) \operatorname{vec}\left(U_{3}\right)^{t}$, obviously $r\left(\tilde{U}_{1 \mid 23}\right)=r\left(\tilde{U}_{12 \mid 3}\right)=1$.

Conversely, if $r\left(\tilde{U}_{1 \mid 23}\right)=1$, there exists an $M \times M$ matrix $U_{1}$ and an $N P \times N P$ matrix $U_{23}$, such that $U=U_{1} \otimes U_{23}$. Therefore

$$
\tilde{U}_{12 \mid 3}=\left(\begin{array}{c}
u_{11} \tilde{U}_{23} \\
u_{12} \tilde{U}_{23} \\
\vdots \\
u_{M M} \tilde{U}_{23}
\end{array}\right),
$$

where $u_{i j}, j=1, \cdots, M$ are the entries of $U_{1} \cdot r\left(\tilde{U}_{12 \mid 3}\right)=1$ if and only if $r\left(\tilde{U}_{23}\right)=1$, that is, there exists an $N \times N$ matrix $U_{2}$ and a $P \times P$ matrix $U_{3}$, such that $U_{23}=U_{2} \otimes U_{3}$, and $U$ is a unitary tensor decomposable matrix.

Let $\rho$ and $\rho^{\prime}$ be two density matrices with orthonormal unitary matrices $X$ and $Y$ as given in (3). Set

$$
V_{0}=X\left(\begin{array}{cccc}
A_{n_{1}} & 0 & \cdots & 0 \\
0 & A_{n_{2}} & \cdots & 0 \\
\vdots & & \ddots & \vdots \\
0 & \cdots & \cdots & A_{n_{r}}
\end{array}\right) Y^{\dagger}
$$

where $n_{i}, i=1,2, \ldots, r$, stands for the geometric multiplicity of the eigenvalue $\lambda_{i}$ of $\rho$, $\sum_{1}^{r} n_{r}=M N P, A_{n_{i}}$ are some unitary $n_{i} \times n_{i}$ complex matrices. The conclusion on bipartite case [9] is still valid, namely, $\rho$ and $\rho^{\prime}$ are equivalent under local unitary transformations if and only if rank $r\left(\tilde{V}_{0}\right)=1$ for some unitary matrices $A_{n_{i}}$. For the case that $\rho$ has distinct eigenvalues, we have

[Theorem 1]. If $\rho$ has distinct eigenvalues, $\rho$ is equivalent to $\rho^{\prime}$ under local unitary transformations if and only if

$$
V=X D Y^{\dagger}
$$

$D=\operatorname{diag}\left(e^{i \theta_{1}}, e^{i \theta_{2}}, \ldots, e^{i \theta_{M N P}}\right)$, contains a unitary tensor decomposable element for some $\theta_{i} \in \mathbb{R}$.

[Proof]. Let $X$ and $Y$ be the unitary matrices that diagonalize $\rho$ and $\rho^{\prime}$ respectively, $\rho=X \Lambda X^{\dagger}, \rho^{\prime}=Y \Lambda Y^{\dagger}$, where $\Lambda=\operatorname{diag}\left(\lambda_{1}, \lambda_{2}, \ldots, \lambda_{M N P}\right)$.

As $\rho$ (resp. $\rho^{\prime}$ ) has distinct eigenvalues, any set $X_{1}$ (resp. $Y_{1}$ ) of unitary eigenvectors corresponding to the eigenvalues of $\rho$ (resp. $\rho^{\prime}$ ) can then be obtained through the following equation: $X_{1}=X U$ (resp. $Y_{1}=Y V$ ), where the unitary matrix $U$ (resp. $V$ ) has the form: $U=\operatorname{diag}\left(e^{i \alpha_{1}}, e^{i \alpha_{2}}, \ldots, e^{i \alpha_{M N P}}\right)$ (resp. $\left.V=\operatorname{diag}\left(e^{i \beta_{1}}, e^{i \beta_{2}}, \ldots, e^{i \beta_{M N P}}\right)\right)$, then $X_{1} D_{1} Y_{1}^{\dagger}=$ $X U D_{1} V^{\dagger} Y^{\dagger}=X D Y^{\dagger}$, where $D_{1}=\operatorname{diag}\left(e^{i \theta_{1}^{\prime}}, e^{i \theta_{2}^{\prime}}, \ldots, e^{i \theta_{M N P}^{\prime}}\right), D=\operatorname{diag}\left(e^{i \theta_{1}}, e^{i \theta_{2}}, \ldots, e^{i \theta_{M N P}}\right)$, $\theta_{i}=\alpha_{i}+\theta_{i}^{\prime}-\beta_{i}, i=1,2, \ldots, M N P$, hence $\rho$ is locally unitary equivalent to $\rho^{\prime}$ if and only if $X D Y^{\dagger}, D=\operatorname{diag}\left(e^{i \theta_{1}}, e^{i \theta_{2}}, \ldots, e^{i \theta_{M N P}}\right)$, contains a unitary tensor decomposable element for some $\theta_{i} \in \mathbb{R}$. 
As an example let us consider a density matrix on $2 \times 2 \times 2$,

$$
\begin{aligned}
\rho & =\left(\begin{array}{cccccccc}
1 & 0 & 0 & 0 & 0 & 0 & 0 & -1 \\
0 & 1 / a & 0 & 0 & 0 & 0 & 0 & 0 \\
0 & 0 & 1 / b & 0 & 0 & 0 & 0 & 0 \\
0 & 0 & 0 & 1 / c & 0 & 0 & 0 & 0 \\
0 & 0 & 0 & 0 & c & 0 & 0 & 0 \\
0 & 0 & 0 & 0 & 0 & b & 0 & 0 \\
0 & 0 & 0 & 0 & 0 & 0 & a & 0 \\
-1 & 0 & 0 & 0 & 0 & 0 & 0 & 1
\end{array}\right) \\
\rho^{\prime} & =\left(\begin{array}{ccccccccc}
1 & 0 & 0 & 0 & 0 & 0 & 0 & 1 \\
0 & a & 0 & 0 & 0 & 0 & 0 & 0 \\
0 & 0 & b & 0 & 0 & 0 & 0 & 0 \\
0 & 0 & 0 & c & 0 & 0 & 0 & 0 \\
0 & 0 & 0 & 0 & 1 / c & 0 & 0 & 0 \\
0 & 0 & 0 & 0 & 0 & 1 / b & 0 & 0 \\
0 & 0 & 0 & 0 & 0 & 0 & 1 / a & 0 \\
1 & 0 & 0 & 0 & 0 & 0 & 0 & 1
\end{array}\right)
\end{aligned}
$$

$\rho^{\prime}$ is in fact a PPT entangled edge state [11]. $\rho^{\prime}$ and $\rho$ have the same eigenvalue set. They are not degenerate in the case $a \neq b \neq c \neq 1$ or 2 or $1 / 2$. Calculating the unitary matrices $X$ and $Y$ that diagonalize $\rho$ and $\rho^{\prime}$, we have $\rho=X \Lambda X^{\dagger}, \rho^{\prime}=Y \Lambda Y^{\dagger}$, where $\Lambda=\operatorname{diag}(2,0,1 / a, a, 1 / b, b, 1 / c, c)$. Denote $D=\operatorname{diag}\left(d_{1}, d_{2}, d_{3}, d_{4}, d_{5}, d_{6}, d_{7}, d_{8}\right)$, we have the matrix $V$ defined by (8),

$$
V=\left(\begin{array}{cccccccc}
\left(-d_{1}+d_{8}\right) / 2 & \left(d_{1}+d_{8}\right) / 2 & 0 & 0 & 0 & 0 & 0 & 0 \\
\left(d_{1}+d_{8}\right) / 2 & \left(-d_{1}+d_{8}\right) / 2 & 0 & 0 & 0 & 0 & 0 & 0 \\
0 & 0 & 0 & d_{2} & 0 & 0 & 0 & 0 \\
0 & 0 & d_{7} & 0 & 0 & 0 & 0 & 0 \\
0 & 0 & 0 & 0 & 0 & d_{3} & 0 & 0 \\
0 & 0 & 0 & 0 & d_{6} & 0 & 0 & 0 \\
0 & 0 & 0 & 0 & 0 & 0 & 0 & d_{4} \\
0 & 0 & 0 & 0 & 0 & 0 & d_{5} & 0
\end{array}\right) .
$$

Therefore

$$
\tilde{V}_{1 \mid 23}=\left(\begin{array}{cccccccccccccccc}
\frac{-d_{1}+d_{8}}{2} & \frac{d_{1}+d_{8}}{2} & 0 & 0 & \frac{d_{1}+d_{8}}{2} & \frac{-d_{1}+d_{8}}{2} & 0 & 0 & 0 & 0 & 0 & d_{2} & 0 & 0 & d_{7} & 0 \\
0 & 0 & 0 & 0 & 0 & 0 & 0 & 0 & 0 & 0 & 0 & 0 & 0 & 0 & 0 & 0 \\
0 & 0 & 0 & 0 & 0 & 0 & 0 & 0 & 0 & 0 & 0 & 0 & 0 & 0 & 0 & 0 \\
0 & d_{3} & 0 & 0 & d_{6} & 0 & 0 & 0 & 0 & 0 & 0 & d_{4} & 0 & 0 & d_{5} & 0
\end{array}\right)
$$




$$
\tilde{V}_{12 \mid 3}=\left(\begin{array}{cccc}
\left(-d_{1}+d_{8}\right) / 2 & \left(d_{1}+d_{8}\right) / 2 & \left(d_{1}+d_{8}\right) / 2 & \left(-d_{1}+d_{8}\right) / 2 \\
0 & 0 & 0 & 0 \\
0 & 0 & 0 & 0 \\
0 & 0 & 0 & 0 \\
0 & 0 & 0 & 0 \\
0 & d_{2} & d_{7} & 0 \\
0 & 0 & 0 & 0 \\
0 & 0 & 0 & 0 \\
0 & 0 & 0 & 0 \\
0 & 0 & 0 & 0 \\
0 & d_{3} & d_{6} & 0 \\
0 & 0 & 0 & 0 \\
0 & 0 & 0 & 0 \\
0 & 0 & 0 & 0 \\
0 & 0 & 0 & 0 \\
0 & d_{4} & d_{5} & 0
\end{array}\right),
$$

obviously, when $d_{1}=-d_{2}=d_{3}=-d_{4}=-d_{5}=d_{6}=-d_{7}=d_{8}=1, r\left(\tilde{V}_{1 \mid 23}\right)=r\left(\tilde{V}_{12 \mid 3}\right)=1$. Hence $\rho$ and $\rho^{\prime}$ are equivalent under local unitary transformations.

Now we consider the multipartite case. A general pure state on $H_{1} \otimes H_{2} \otimes \ldots \otimes H_{M}$ is of the form

$$
\left|\Psi_{M}\right\rangle=\sum_{k=1}^{M} \sum_{i_{k}=1}^{N_{k}} a_{i_{1} i_{2} \ldots i_{M}}\left|e_{i_{1}}\right\rangle \otimes\left|f_{i_{2}}\right\rangle \otimes \ldots \otimes\left|g_{i_{M}}\right\rangle, \quad a_{i_{1} i_{2} \ldots i_{M}} \in \mathbb{C}
$$

with $\sum a_{i_{1} i_{2} \ldots i_{M}} a_{i_{1} i_{2} \ldots i_{M}}^{*}=1,\left|e_{i_{1}}\right\rangle,\left|f_{i_{2}}\right\rangle, \ldots,\left|g_{i_{M}}\right\rangle, i_{k}=1,2, \ldots, N_{k}, k=1,2, \ldots, M$, the corresponding orthonormal basis of complex Hilbert spaces $H_{1}, H_{2}, \ldots, H_{M}$. Two density matrices $\rho$ and $\rho^{\prime}$ are said to be equivalent under local unitary transformations if there exist unitary operators $U_{1}$ on $H_{1}, U_{2}$ on $H_{2}, \cdots$, and $U_{M}$ on $H_{M}$ such that $\rho^{\prime}=\left(U_{1} \otimes U_{2} \otimes \cdots \otimes\right.$ $\left.U_{M}\right) \rho\left(U_{1} \otimes U_{2} \otimes \cdots \otimes U_{M}\right)^{\dagger}$. For any non-degenerate density matrices we have

[Theorem 2]. Let $\rho$ and $\rho^{\prime}$ be two non-degenerate density matrices on $H_{1} \otimes H_{2} \otimes \ldots \otimes H_{M}$ and $X$ and $Y$ the unitary matrices that diagonalize $\rho$ and $\rho^{\prime}$ respectively, $\rho=X \Lambda X^{\dagger}, \rho^{\prime}=$ $Y \Lambda Y^{\dagger}$, where $\Lambda=\operatorname{diag}\left(\lambda_{1}, \lambda_{2}, \ldots, \lambda_{N_{1} N_{2} \cdots N_{M}}\right) . \rho$ and $\rho^{\prime}$ are equivalent under local unitary transformations if and only if the $N_{1} N_{2} \cdots N_{M} \times N_{1} N_{2} \cdots N_{M}$ unitary matrix $V=X D Y^{\dagger}$, $D=\operatorname{diag}\left(e^{i \theta_{1}}, e^{i \theta_{2}}, \ldots, e^{i \theta_{N_{1} N_{2} \cdots N_{M}}}\right)$, satisfies

$$
r\left(\tilde{V}_{1 \mid 2 \cdots M}\right)=r\left(\tilde{V}_{12 \mid 3 \cdots M}\right)=\cdots=r\left(\tilde{V}_{12 \cdots M-1 \mid M}\right)=1,
$$

where

$$
\begin{aligned}
& \left(\tilde{V}_{1 \mid 2 \cdots M}\right)_{i_{1} i_{1}^{\prime}, i_{2} \cdots i_{M} i_{2}^{\prime} \cdots i_{M}^{\prime}}=(V)_{i_{1} i_{2} \cdots i_{M}, i_{1}^{\prime} i_{2}^{\prime} \cdots i_{M}^{\prime}}, \\
& \left(\tilde{V}_{12 \mid 3 \cdots M}\right)_{i_{1} i_{2} i_{1}^{\prime} i_{2}^{\prime}, i_{3} \cdots i_{M} i_{3}^{\prime} \cdots i_{M}^{\prime}}=(V)_{i_{1} i_{2} \cdots i_{M}, i_{1}^{\prime} i_{2}^{\prime} \cdots i_{M}^{\prime}}, \\
& \cdots \cdots \cdots \cdots \\
& \left(\tilde{V}_{12 \cdots M-1 \mid M}\right)_{i_{1} i_{2} \cdots i_{M-1} i_{1}^{\prime} i_{2}^{\prime} \cdots i_{M-1}^{\prime}, i_{M} i_{M}^{\prime}}=(V)_{i_{1} i_{2} \cdots i_{M}, i_{1}^{\prime} i_{2}^{\prime} \cdots i_{M}^{\prime}} .
\end{aligned}
$$

We have studied the equivalence of multipartite quantum mixed states under local unitary transformations in terms of analysis of fixed point subgroup and tensor decomposability of certain matrices. A criterion for the equivalence of non-degenerate mixed multipartite 
quantum mixed states under local unitary transformations has been presented. In fact this

approach works for general multipartite mixed states. But then in stead of the rank $r(\tilde{V})$, one has to verify if $r\left(\tilde{V}_{0}\right)$ could be one for all possible matrices $A_{n_{i}}$, which is again complicated. The problem is dramatically simplified when the degeneracy of the related density matrices is reduced. In particular, for the non-degenerate case, two density matrices are easily verified whether they are equivalent or not under local unitary transformation.

Acknowledgments The first and last named author gratefully acknowledges the support provided by the China-Germany Cooperation Project 446 CHV 113/231, "Quantum information and related mathematical problems".

\section{References}

[1] E.M. Rains, IEEE Transactions on Information Theory 46, 54 (2000).

[2] M. Grassl, M. Rötteler, and T. Beth, Phys. Rev. A 58, 1833 (1998).

[3] Y. Makhlin, Quant. Info. Proc. 1, 243 (2002).

[4] N. Linden, S. Popescu, and A. Sudbery, Phys. Rev. Lett. 83, 243 (1999).

[5] S. Albeverio, S.M. Fei, P. Parashar, and W.L. Yang, Phys. Rev. A 68, 010313 (R) (2003).

[6] S. Albeverio, S.M. Fei and D. Goswami, Local Invariants for a Class of Mixed States, SFB-611 preprint, 2004.

[7] S. Albeverio, L. Cattaneo, S.M. Fei and X.H. Wang, Equivalence of Tripartite Quantum States under Local Unitary Transformations, to appear in Int. J. Quant. Inform. (2005).

[8] Sergio Albeverio, Laura Cattaneo, Shao-Ming Fei and Xiao-Hong Wang, Multipartite States under Local Unitary Transformations, SFB-611 preprint, 2004.

[9] S.M. Fei and N.H. Jing, Equivalence of Quantum States under Local Unitary Transformations, to appear in Phys. Lett. A(2005).

[10] N.P. Pitsianis, Ph.D. thesis, The Kronecker Product in Approximation and Fast Transform Generation, Cornell University, New York (1997).

[11] A. Acín, D. Bruß, M. Lewenstein and A.Sanpera, Phys. Rew. Lett. 87, 040401 (2001). 